\title{
PROMOVE-Crianças: efeitos de um treino em habilidades sociais para crianças com problemas de comportamento
}

\section{PROMOVE-Children: effects of training in social skills for children with behavior problem}

PROMOVE-niños: efectos de un entrenamiento en habilidades
sociales para niños con problemas de conducta

\section{Alessandra Pereira Falcão*}

Universidade Estadual Paulista - Julio de Mesquita Filho - UNESP, Bauru, São Paulo, Brasil

\section{Alessandra Turini Bolsoni-Silva**}

Universidade Estadual Paulista - Julio de Mesquita Filho - UNESP, Bauru, São Paulo, Brasil

\section{Natália Magri***}

Universidade Estadual Paulista - Julio de Mesquita Filho - UNESP, Bauru, São Paulo, Brasil

\section{Laísa Aparecida Moretto****}

Universidade Estadual Paulista - Julio de Mesquita Filho - UNESP, Bauru, São Paulo, Brasil

\begin{abstract}
RESUMO
O objetivo do estudo foi avaliar a eficácia de uma intervenção em grupo com crianças para promover habilidades sociais infantis e reduzir problemas de comportamento. Os participantes foram sete crianças: duas meninas e cinco meninos, com idades entre 7 e 9 anos, que frequentavam o segundo ano do ensino fundamental. Foram diagnosticados como clínicas para problemas de comportamento, através dos instrumentos CBCL e TRF respondidos por pais e professores respectivamente, antes e após a intervenção, em quatro fases: linha de base; pré-teste; pós-teste e seguimento, como medida de resultado. A intervenção constou de oito sessões em que foram ensinadas habilidades sociais. Como medida de processo foram analisadas as filmagens de todas as sessões, organizadas em iniciais, intermediárias e finais. Os problemas de comportamento mantiveram-se em baixa frequência, durante toda a intervenção, e as habilidades aumentaram de frequência. A partir do CBCL e TRF pode-se verificar diferença estatística significativa quanto aos comportamentos externalizantes nas medidas de pré e pós-teste. $O$ procedimento foi capaz de ensinar habilidades sociais que mudaram estatisticamente quando comparadas as fases inicial-intermediária e
\end{abstract} intermediária-final. 
Palavras-chave: problemas de comportamento, habilidades sociais, intervenção em grupo.

\begin{abstract}
The objective of the study was to evaluate the effectiveness of a group intervention with children to promote children's social skills and reduce behavior problems. Participants were seven children (two girls and five boys, aged between 7 and 9 years) attending the second year of elementary school and were diagnosed as clinical tools for the CBCL behavior problems and TRF answered by parents and teachers respectively, before and after the intervention, in four phases (baseline, pre-test, post-test and monitoring), as a result measure. The intervention consisted of eight sessions in which social skills were taught. As process as the recordings were analyzed for all sessions, organized in the early, intermediate and final. Behavior problems remained at low frequency throughout the intervention From the $\mathrm{CBCL}$ and TRF can be verified statistically significant difference in the externalizing behaviors when compared stages of pre and post-test. The procedure was able to teach social skills that have changed statistically compared the initial-intermediate and intermediate-final stage.
\end{abstract}

Keywords: behavior problems, social skills, group intervention.

\begin{abstract}
RESUMEN
El objetivo del estudio fue evaluar la eficacia de una intervención grupal con los niños para promover las habilidades sociales de los niños y reducir los problemas de comportamiento. Los participantes fueron siete hijos (dos niñas y cinco niños, de edades comprendidas entre los 7 y 9 años) que asisten al segundo año de la escuela primaria y se diagnosticaron como herramientas clínicas para los problemas de conducta CBCL y TRF respondidas por los padres y maestros, respectivamente, antes de y después de la intervención, en cuatro fases (línea de base, antes de la prueba, después de la prueba y monitoreo), como medida de resultado. La intervención consistió en ocho sesiones en las que se enseñan habilidades sociales. Como proceso, se analizaron las grabaciones de todas las sesiones, organizadas en el temprano, intermedio y final. Los problemas de conducta se mantuvieron en baja frecuencia a lo largo de la intervención Desde el CBCL y TRF puede verificarse diferencias estadísticamente significativas en las conductas de externalización en comparación etapas de pre y post-test. El procedimiento fue capaz de enseñar habilidades sociales que han cambiado en comparación estadísticamente la etapa inicial intermedia e intermedia final.
\end{abstract}

Palabras-clave: problemas de conducta, habilidades sociales, de intervención grupal.

\title{
1 I ntrodução
}

A intervenção em habilidades sociais na infância tem sido defendida como forma de reduzir problemas de comportamento (Kim, Doh, Hong \& Choi, 2010; Webster-Stratton, Reid, \& Hamond, 2001), com resultados positivos. No entanto, são poucos os estudos que focalizam o ensino de habilidades sociais, considerando a psicologia preventiva indicada (Scott, Knapp, Henderson, \& Maughan, 2001) e a psicologia baseada em evidência (American Psychological Association 
[APA], 2006), de forma a promover intervenções que sejam simultaneamente eficazes (que atinjam os objetivos iniciais) e efetivas (de curto prazo e com baixos custos), isto é, que garantam os resultados esperados no menor tempo possível (Cox, 2005).

Estudos em psicologia preventiva têm afirmado que crianças com comportamentos anti-sociais e/ou desvios de conduta, quando não passam por intervenção precocemente, podem se tornar adultos com problemas de interação social (Scott, Knapp, Henderson, \& Maughan, 2001). Desse modo, a prevenção indicada é de grande importância na capacitação dos indivíduos para que não se comportem de maneira prejudicial no futuro (Oliveira, 2012). Procedimentos que ensinam habilidades sociais são modelos de prevenção já que a aprendizagem dessas habilidades age como um fator de proteção ao surgimento de problemas de comportamento (Oliveira, 2012).

O estudo de habilidades sociais em crianças justifica-se, pois a literatura tem encontrado relação inversa entre esse repertório e problemas de comportamento (Berry \& O'Connor, 2010; BolsoniSilva \& Loureiro, 2011; Bolsoni-Silva, Marturano \& Loureiro, 2011; Barham \& Cia, 2009; Kim et al, 2010; Leme \& Bolsoni-Silva, 2010a). No entanto não há consenso sobre tal relação, como visto em Farmer e Xie (2007) o que sinaliza a importância de novas pesquisas com essa temática.

Se habilidades sociais e problemas de comportamento podem estar empiricamente relacionados, uma explicação possível, a partir de Goldiamond (2002/1974), é a de que os comportamentos socialmente habilidosos podem ser funcionalmente equivalentes a problemas de comportamento (Bolsoni-Silva \& Carrara, 2010), ou seja, se a criança usar das habilidades sociais para obter atenção e resolver problemas, pode reduzir a probabilidade de apresentar problemas de comportamento. Esses, por sua vez, podem ser considerados como déficits ou excessos comportamentais que prejudicam o acesso a novas contingências de reforçamento (BolsoniSilva, 2003). Topograficamente podem ser classificados como externalizantes, por exemplo, agressividade e desobediência e internalizantes, tais como ansiedade, depressão e queixas somáticas (Achenbach \& Edelbrock, 1979). Muitos pesquisadores têm relatado que tais dificuldades trazem impacto para as interações sociais e/ou desempenho acadêmico da criança (Bolsoni-Silva, Paiva \& Barbosa, 2009; Maravieski \& Serralta, 2011; Wielewichi, 2011).

Vários autores apresentaram categorias de habilidades sociais importantes para o desenvolvimento infantil, entre eles, A. Del Prette e Del Prette (2005): "autocontrole e expressividade emocional", "civilidade", "empatia", "assertividade", "fazer amizades", "solução de problemas interpessoais" e "habilidades sociais acadêmicas". BolsoniSilva, Marturano, Pereira e Manfrinato (2006): "disponibilidade social e cooperação", "expressão de sentimentos e enfrentamento" e 
"interação social positiva". Bolsoni-Silva, Marturano e Loureiro (2009): "fator sociabilidade", "fator iniciativa" e "fator busca de suporte".

A ideia de ensinar habilidades sociais para reduzir problemas de comportamento foi implementada por alguns autores. WebsterStratton et al. (2001) afirmaram que após o tratamento em habilidades sociais, as crianças do grupo de intervenção apresentaram menos problemas de externalização em casa e menos comportamentos agressivos na escola, eram mais pró-sociais com os pares, além de utilizarem mais estratégias em situações de conflitos do que as crianças do grupo controle. Kim et al (2010) apontaram que as crianças que passaram pela intervenção em habilidades sociais apresentaram menor frequência de comportamento agressivo e melhoria no comportamento pró-social, regulação emocional e habilidades sociais. Herman, Borden, Reinke e Webster-Stratton (2011) também encontraram que a intervenção em habilidades sociais com as crianças reduziu problemas de comportamento na comparação com o grupo controle. Essas pesquisas, conduzidas no exterior, tiveram controle experimental e número grande de participantes, no entanto, o termo habilidades sociais é usado como sinônimo de pró-social e os comportamentos ensinados não necessariamente são os mesmos, considerando cada um dos estudos, e também não correspondem às diversas classes de habilidades sociais documentadas, por exemplo, por Bolsoni-Silva et al. (2009).

No Brasil, estudos de intervenção em habilidades sociais vêm sendo realizados com objetivos mais focais e com um número menor de participantes. A maioria dos estudos de intervenção encontrados na literatura, para o tratamento de problemas de comportamento, envolve intervenções em habilidades sociais com pais (Coelho \& Murta, 2007; Kanamota, 2013; Lambertucci \& Carvalho, 2008; Orti, Bolsoni-Silva, Grecco, \& Matsunaka, 2015; Orti, Bolsoni-Silva, \& Villa, 2015; Pinheiro, Haase, A. Del Prette, Amarante, \& Del Prette, 2006) ou com pais e crianças (Gonçalves \& Murta, 2008; Salvo, Mazzarotto, \& Lörh, 2005). Intervenções que focam apenas em promover habilidades específicas, como resolução de problemas (Elias, Marturano, \& Motta-Oliveira, 2012) ou empatia (Vettorazzi et al., 2005) e envolvem um grande número de sessões a serem realizadas. Essas pesquisas deixam de ensinar uma classe maior de habilidades sociais e necessitam de muitas sessões para sua condução. Além disso, a maioria das pesquisas encontradas com intervenção em habilidades sociais com crianças, apresentam dados apenas de pré e pós-teste (Elias, Marturano, \& Motta-Oliveira, 2012; Vettorazzi et al., 2005), o que dificulta afirmar que os repertórios clínicos das crianças seriam mantidos se não tivesse ocorrido a intervenção. Outro aspecto a ser destacado, é o uso da avaliação processual do desempenho dos participantes. Em revisão bibliográfica realizada foram encontrados 
poucos estudos com intervenção em habilidades sociais com crianças (Borges \& Marturano, 2003; Castro, Melo, \& Silvares, 2003; Ferreira \& Del Prette, 2013; Lopes, A. Del Prette, \& Del Prette, 2013) que priorizassem a descrição dos comportamentos apresentados pelas crianças durante o processo em relação aos resultados.

A grande maioria dos estudos (Coelho \& Murta, 2007; Elias, Marturano, \& Motta-Oliveira, 2012; Gonçalves \& Murta, 2008; Lambertucci \& Carvalho, 2008; Salvo, Mazzarotto, \& Lörh, 2005; Vettorazzi et al., 2005) valoriza a avaliação do desempenho com instrumentos pré e pós intervenção, não apontando as mudanças dos participantes ao longo da intervenção, o que acredita-se ser de grande importância (Tourinho et al, 2007; Wampold, Goodheart, \& Levant, 2007). São encontrados ainda estudos que apresentam modelos de intervenção mas sem avaliar a efetividade (Comodo, A. Del Prette, Del Prette, \& Manólio, 2011), ou na forma de estudo de caso, que ainda que traga informações relevantes, tem limitações metodológicas (Branco \& Ferreira, 2006); estudos de revisão ou não especificam claramente quais intervenções são com crianças (Machado, Dahl, Carvalho, \& Cavalcanti, 2007) ou então afirmam que intervenções apenas com as crianças com problemas de comportamento são escassas na literatura PSI, havendo sobretudo estudos combinados com pais, professores e crianças (Bolsoni-Silva, Villas Boas, Leme, \& Silveira, 2010).

No caso do presente texto, pretende-se descrever resultados de um procedimento de intervenção que ateste sua efetividade para tratar problemas de comportamento a partir do ensino de habilidades sociais infantis, de forma a tratar problemas presentes e prevenir dificuldades futuras (Weisz, Sandler, Durlak, \& Anton, 2005), sendo a tônica atual da psicologia preventiva, em que tratamento e prevenção fazem parte simultaneamente do modelo (Oliveira, 2012).

Com o objetivo de realizar um procedimento com crianças considerando a psicologia baseada em evidência e a psicologia preventiva é que se elaborou um procedimento de intervenção com crianças que busca: ensinar habilidades sociais que discriminem empiricamente problemas de comportamento (Leme \& Bolsoni-Silva, 2010a), as quais aumentam a probabilidade de obter reforçadores naturais, inclusive no grupo clínico (Leme \& Bolsoni-Silva, 2010b). O trabalho completo inclui medidas de linha de base, pré-teste, pósteste e seguimento, valendo-se de relato e de observação direta. Dessa forma, o objetivo é descrever a forma pela qual se ensinou habilidades sociais para crianças a partir de um procedimento de intervenção, considerando: (a) medidas de produto (relato), em avaliações de linha de base, pré-teste, pós-teste e seguimento e (b) medidas de processo (observação direta): problemas de comportamento e habilidades sociais durante as sessões de intervenção. 


\section{Método}

\subsection{Aspectos éticos}

A pesquisa foi submetida ao Comitê de Ética em Pesquisa e obteve o parecer favorável.

\subsection{Delineamento}

Trata-se de um delineamento de sujeito único, com medidas de linha de base, pré e pós-testes e seguimento (Cozby, 2006). Para avaliação do processo são apresentados os comportamentos de cada participante ao longo de cada sessão de intervenção. Para medidas de produto são apresentadas as frequências da somatória dos comportamentos apresentados pelo grupo de crianças nos instrumentos selecionados (CBCL e TRF) e uma tabela com os participantes clínicos em cada fase da avaliação.

\subsection{Participantes}

Participaram do estudo sete crianças (duas meninas e cinco meninos) clínicas para problemas de comportamento internalizantes, externalizantes e totais em dois contextos (escola e família), ou seja, eram crianças que tinham comorbidade de problemas internalizantes e externalizantes, cujos problemas foram identificados tanto por pais como por professoras. As crianças frequentavam o segundo ano do ensino fundamental (tinham entre sete e nove anos) de uma escola municipal de uma cidade do interior paulista. Somente duas das crianças participantes eram colegas de sala. Seis (85\%) dos participantes tinham 7 anos e um (15\%) 9 anos. Também participaram pais e professoras das crianças que responderam instrumentos de relato acerca dos comportamentos delas.

\subsection{I nstrumentos}

CBCL "Child Behavior Checklist" e TRF "Teachers Report Form" (Inventários de Comportamentos da Infância e Adolescência, Achenbach \& Rescorla, 2001) para pré-escolares e escolares (4 a 18 anos) que investigam, respectivamente a partir do relato de pais e de professores, a frequência de 113 respostas indicativas de problemas de comportamento. Os resultados são organizados de acordo com as escalas de problemas internalizantes, externalizantes e totais.

Protocolo de observação, que continha as habilidades sociais (A. Del Prette, \& Del Prette, 2005; Gonçalves \& Murta, 2008) e os problemas de comportamento (Barham \& Cia, 2009; Leme \& Bolsoni-Silva, 
2010a). Esse instrumento foi elaborado para a avaliação processual do desempenho dos participantes a partir de filmagens.

\subsection{Procedimento de intervenção}

A intervenção em Habilidades Sociais foi elaborada pela pesquisadora e consistiu em oito sessões (Falcão \& Bolsoni-Silva, 2016). As habilidades sociais foram selecionadas a partir de estudos de comparações de grupo encontrados na literatura brasileira, para que assim os comportamentos ensinados estejam diretamente ligados à realidade dos Participantes (Berry \& O'Connor, 2010; Bolsoni-Silva, Marturano, Pereira, \& Mafrinato, 2006; Kim et al., 2010; Leme \& Bolsoni-Silva, 2010; Leme, 2008) e/ou correlacionais (Barham \& Cia, 2009; Cia \& Barham, 2009; Elias, Marturano \& Motta-Oliveira, 2012; Herman et al., 2011). As sessões aconteciam semanalmente, eram realizadas com grupos de cinco crianças. Elas duravam cerca de uma hora e meia e eram realizadas no contra-turno do período escolar dos participantes em uma sala localizada na própria escola.

Para a descrição das habilidades sociais durante as sessões do procedimento de intervenção foram selecionados trechos de uma animação (Tinker Bell e o Tesouro Perdido, 2009) a partir de revisão de literatura que identificou habilidades sociais que diferenciavam crianças com e sem problemas de comportamento. Os trechos eram passados ao início de cada sessão de modo que os comportamentos apresentados pelas personagens fossem acompanhados de modo integral ao longo das sessões. Era realizada a análise funcional dos comportamentos apresentados pelas personagens e após a discussão inicial as crianças participavam de atividades que buscavam aproximar as habilidades sociais apresentadas aos seus contextos. As atividades incluíam: elaboração de estórias a partir de fantoches, colagem de situações, role-playing de situações, atividades com bexigas e discussão de situações do cotidiano. Adicionalmente, a contextualização do ambiente, a partir de estímulos antecedentes, comportamentos e suas consequências foram facilitadoras da análise de contingências realizada pela aplicadora juntamente com as crianças, bem como da modelagem de diversas habilidades sociais, daquelas que constam propriamente no treino direto e de outras, tais como 'pedir permissão', 'contar fatos', 'falar coisas engraçadas', 'interagir de forma não verbal', 'dar sugestões'. Dessa forma destacase que a psicóloga reforçava diferencialmente os comportamentos apresentados pelos participantes, buscando aumentar a frequência de habilidades sociais e reduzir problemas de comportamento. Em um segundo momento, foram realizadas atividades lúdicas que buscavam treinar as habilidades analisadas. Em cada uma das sessões era desenvolvida uma atividade diferente. As habilidades ensinadas durante as sessões foram: sessão 1- cumprimentar, iniciar conversas, 
civilidade; sessão 2- agradecer, falar coisas boas, expressar opiniões; sessão 3- fazer amigos, ajudar, brincar, dividir suas coisas; sessão 4esperar a minha vez, me controlar; sessão 5- fazer e atender pedidos; sessão 6- nomear sentimentos, empatia; sessão 7- elogiar, beijar, abraçar; sessão 8- admitir erros, pedir desculpas e ouvir críticas.

\subsection{Procedimento de coleta de dados}

Após a aprovação da secretaria de educação da cidade em que foram colhidos os dados, as crianças foram encaminhadas pelos professores de sala de aula regular do segundo ano do ensino fundamental. $\mathrm{Na}$ sequência o TRF confirmou diagnóstico e, então, os pais foram contatados para responderem o $\mathrm{CBCL}$, o qual também confirmou problemas de comportamento nas escalas de internalizantes, externalizantes e problemas totais. Professores, pais e alunos assinaram respectivamente o Termo de consentimento Livre e Esclarecido (TCLE), e as sessões de intervenção foram filmadas, também com a autorização dos pais.

\subsection{Procedimento de análise de dados}

Quanto à observação das filmagens, o procedimento utilizado incluiu a avaliação por três observadores independentes (O1, O2 e 03), que após treino em que foram ensinados a cada um dos observadores as topografias de cada um dos comportamentos, após o ensino os observadores tabularam uma sessão escolhida até que chegassem à índices confiáveis de concordância (Fagundes, 1999): O1 e O2=0,88, $\mathrm{O} 1$ e $\mathrm{O} 3=0,90, \mathrm{O} 2$ e $\mathrm{O} 3=0,98$.

Todas as oito sessões de intervenção foram analisadas na íntegra, ou seja, do momento em que as crianças entravam na sala para a intervenção até o momento em que se despediam e se retiravam da sala. O critério de registro, a título de exemplo, foi: se a criança fazia perguntas dos minutos 1 ao 2 , era registrado como um comportamento de fazer perguntas; se ela não fizesse perguntas dos minutos 2 a 3 nada era registrado para aquele comportamento; e se ela voltasse a fazer perguntas dos minutos 3 a 4 era registrado.

Para o tratamento de dados descritivo e inferencial, os problemas de comportamento e as habilidades sociais foram somados e organizados por sessões, em um primeiro momento. Na sequência as sessões foram agrupadas em inicial ( 1 e 2), intermediárias ( 3,4 e 5 ) e finais $(6,7$ e 8$)$, para que comparações não paramétricas (teste Wilcoxon) fossem conduzidas entre início-intermediário, intermediário-final e início-final. Os resultados estão organizados no corpo do texto e em Figuras. 
Quanto aos instrumentos CBCL e TRF, foram corrigidos de acordo com os manuais padronizados que fornecem como resultados 0 diagnóstico clínico, não-clínico e limítrofe para problemas de comportamento internalizantes, externalizantes e totais para as crianças e foram realizadas comparações, por escores, não paramétricas (teste Wilcoxon) entre linha de base, pré-teste, pósteste e seguimento. Foram consideradas clínicas as crianças que pontuaram nas escolas gerais de problemas externalizantes, e/ou internalizantes e/ou total de problemas.

\section{Resultados}

Nessa seção são apresentados três conjuntos de informações: (a) Medidas de produto: as variáveis com diferença estatisticamente significativa entre os instrumentos CBCL e TRF aplicados em pais e professores na linha de base, pré, pós-teste e seguimento dos comportamentos apresentados durante as sessões de intervenção (descritos no corpo do texto); (b) Medidas de processo: tratamento estatístico da frequência total de comportamentos apresentados pelas crianças durante as sessões de intervenção de grupo, organizadas em iniciais, intermediárias e finais; e (c) Medidas de processo: descrição da frequência de comportamentos dos participantes, em cada uma das sessões (Figuras 1 e 2 ).

\subsection{Medidas de produto}

Quando comparadas as fases de linha de base, pré-teste, pós-teste e seguimento, tanto do instrumento $\mathrm{CBCL}$ respondido pelos pais das crianças participantes, como do instrumento TRF, respondido pelas professoras dos participantes somente a variável comportamento externalizante apresentou diferença estatística com significância (para o CBCL $p=0,027, z=-2,214$ e para o TRF $p=0,058, z=-$ $1,897)$. No CBCL foram apresentadas as seguintes as médias dos escores dos comportamentos externalizantes, nas fases de pré ( édia $=73,00$ ) e pós-teste (média $=70,00$ ), a redução da frequência de comportamento quando comparada essas duas fases apresentou $z=-2,214$ e $p=0,027$ considerada estatisticamente significativa. Quando comparadas as médias dos escores dos comportamentos externalizantes apresentados nas demais fases, linha de base (média $=72,85)$ e pré-teste (média $=73,00$ ) e pós-teste ( média $=70,00$ ) e seguimento (média $=71,50$ ) não houve diferença significativa estatisticamente (LB/pré- $p=1,00, z=000$ e pós/seg $p=0,593, z=0,536)$. Para o TRF, quando comparadas as fases de pré (média $=74,29$ ) e pós-teste (média $=69,00$ ), indicando que esses comportamentos foram emitidos em menor frequência pelos 
participantes após a intervenção. Também para esse instrumento quando comparadas as fases de linha de base (média $=69,57$ ) e préteste (média $=74,58$ ) e pós-teste (média $=69,00$ ) e seguimento (média $=56,50$ ) não houve diferença significativa estatisticamente (LB/pré $p=0,180, z=-1,342$ e pós/seg $p=0,144, z=-1,461$ ). As variáveis comportamentos internalizantes e totais dos dois instrumentos ( $\mathrm{CBCL}$ e TRF) não apresentaram diferença estatística com significância quando comparadas as quatro fases.

Tabela 1

Participantes com escores clínicos nas medidas de avaliação

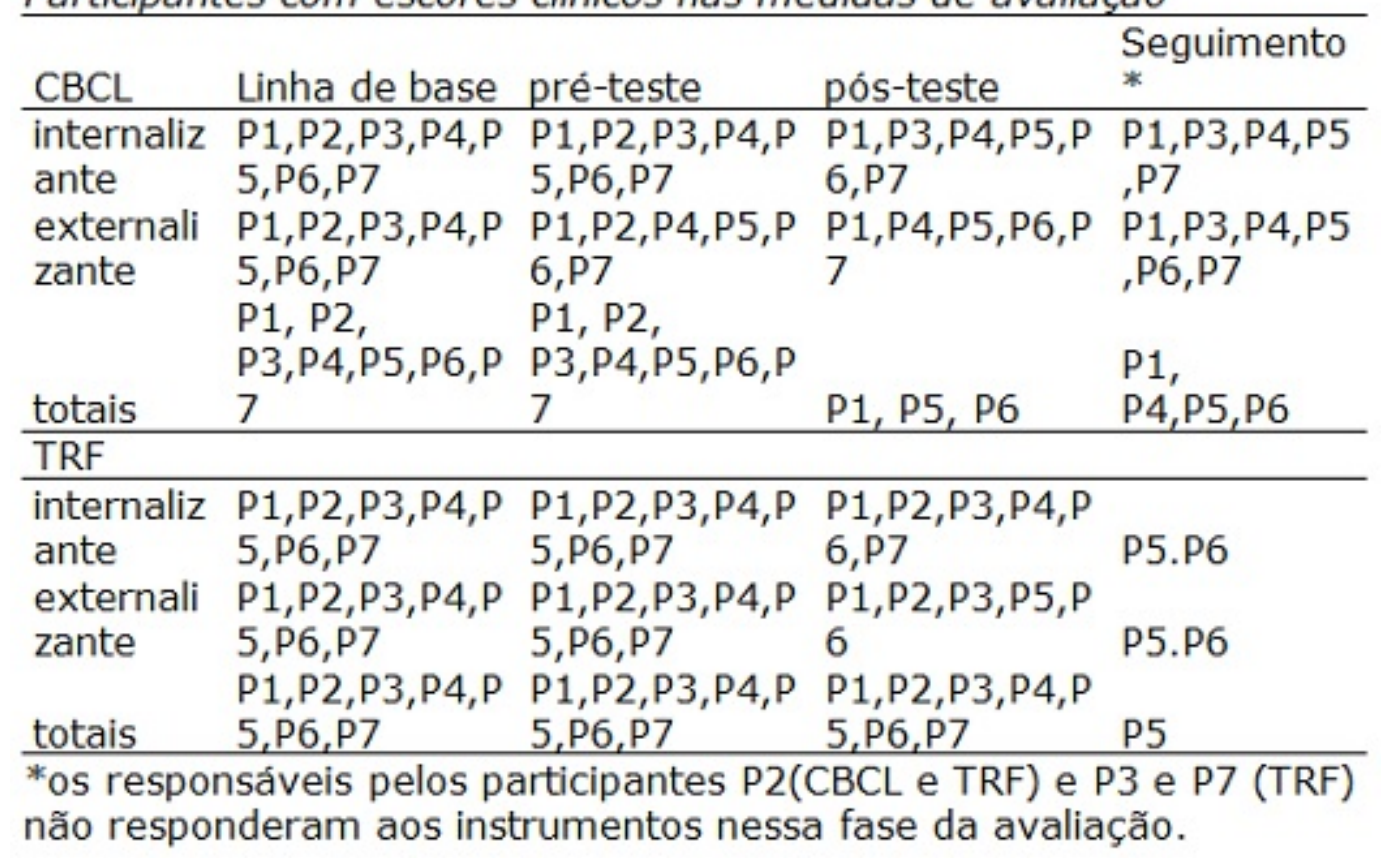

Os dados apontam que quanto aos comportamentos internalizantes do CBCL P2 saiu do nível clínico no pós-teste e P6 no seguimento. Quanto aos comportamentos externalizantes P2 e P3 saíram do nível clínico no pós-teste. Quanto aos comportamentos totais P2, P3, P4 e P7 saíram dos níveis clínicos no pós-teste e P3 e P7 se mantiveram no seguimento. Já para o TRF quanto aos comportamentos internalizantes P5 saiu do nível clínico no pós-teste e P1 e P4 no seguimento. Quanto aos comportamentos externalizantes P4 e P7 saíram do nível clínico no pós-teste e P1, P4 e P5 no seguimento. Quanto aos comportamentos totais P1, P4 e P6 saíram dos níveis clínicos no seguimento.

\subsection{Medidas de processo}

A ocorrência das habilidades sociais das crianças, sessão a sessão, encontra-se na Figura 1. De acordo com a Figura 1 nota-se alta frequência de habilidades sociais emitidas pelas crianças 
(porcentagem média de 94,76\%), a qual aumentou no decorrer da intervenção. O tratamento estatístico revelou diferenças entre as sessões do início e as intermediárias (mediana início $=101,14$; mediana intermediário $=181,14, p=0,018$ ) e entre início e final ( mediana $=101,14 ;$ mediana $=176,00, p=0,018)$ das habilidades sociais apresentadas pelas crianças durante as sessões de intervenção. Quando comparadas as fases intermediária e final ( mediana $=181,14 ;$ mediana $=176,00, p=0,352$ ) não houve diferença significativa. Quanto aos problemas de comportamento, de acordo com a Figura 2, a frequência foi muito baixa durante todo o procedimento $(5,24 \%)$, e não teve mudança significativa no decorrer das sessões.

Quando comparadas as médias das habilidades sociais e problemas de comportamento apresentados nas três fases (início, meio e final) pode-se verificar que os comportamentos da categoria de habilidades sociais "disponibilidade social e cooperação" foi a que mais ocorreu durante as sessões de intervenção nas três fases de início $(93,36 \%)$, meio $(96,03 \%)$ e final $(96,99 \%)$. Os comportamentos que envolviam essa categoria eram o foco da intervenção, já que foram apontados pela literatura (Barham \& Cia, 2009; Bolsoni-Silva, Marturano, Pereira, \& Mafrinato, 2006; Cia \& Barham, 2009; Elias, Marturano, \& Motta-Oliveira, 2012; Leme \& Bolsoni-Silva, 2010) da área como os diferenciadores entre os repertórios de crianças clínicas e não-clínicas para problemas de comportamento e/ou correlacionais. Exemplos de comportamentos dessa categoria são comportamentos de civilidade que envolvem cumprimentar as pessoas, o que ocorria com facilidade em todas as sessões. Outro exemplo seria participar das atividades propostas e se controlar para prestar atenção ao filme. Os comportamentos das outras categorias de habilidades sociais aconteceram em baixa frequência durante as sessões. 

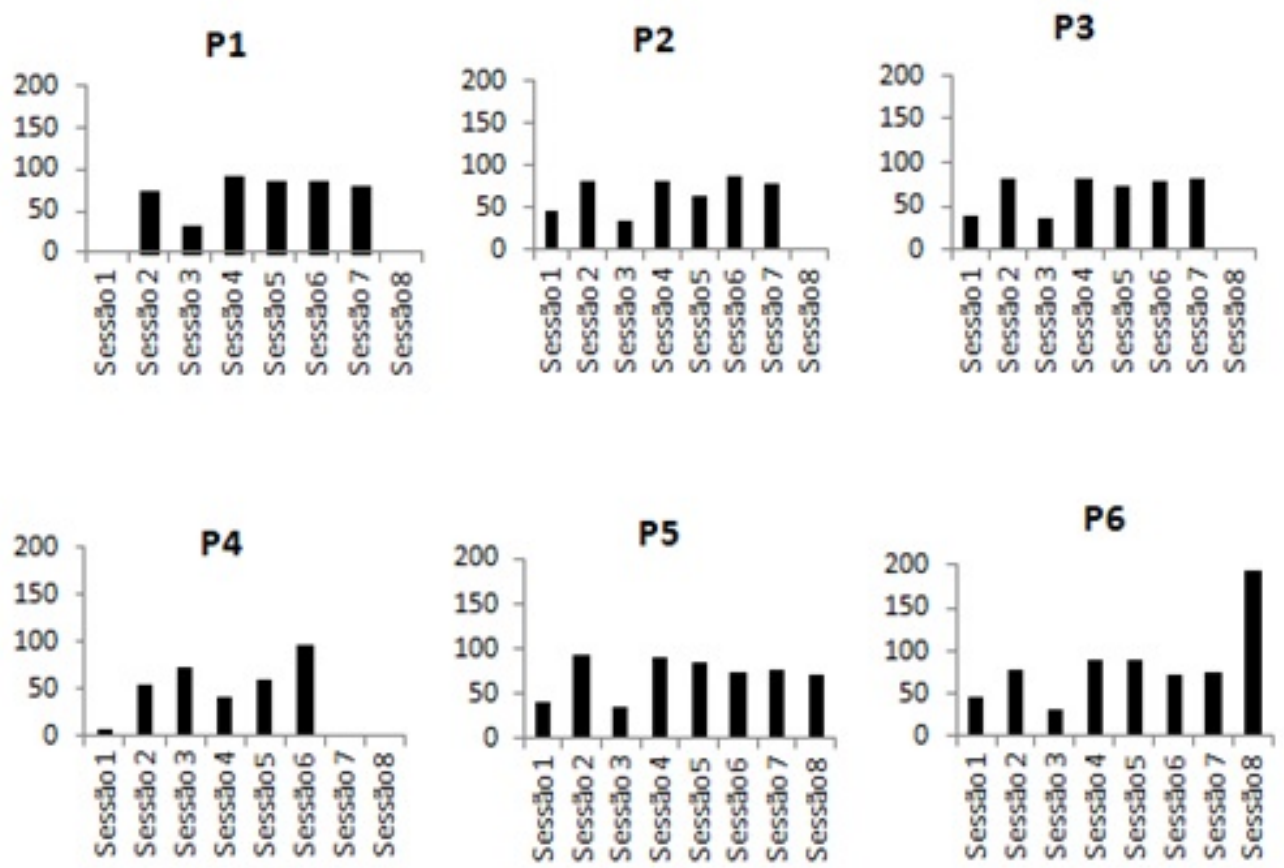

P7

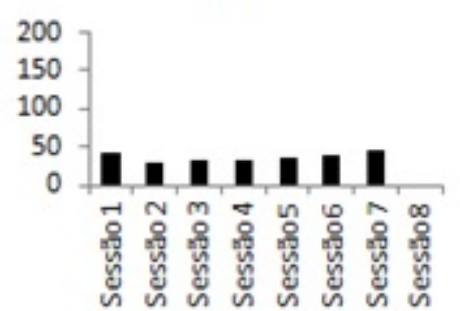

Figura 1. Frequência de habilidades sociais apresentadas por cada um dos participantes em cada uma das sessões de intervenção. 

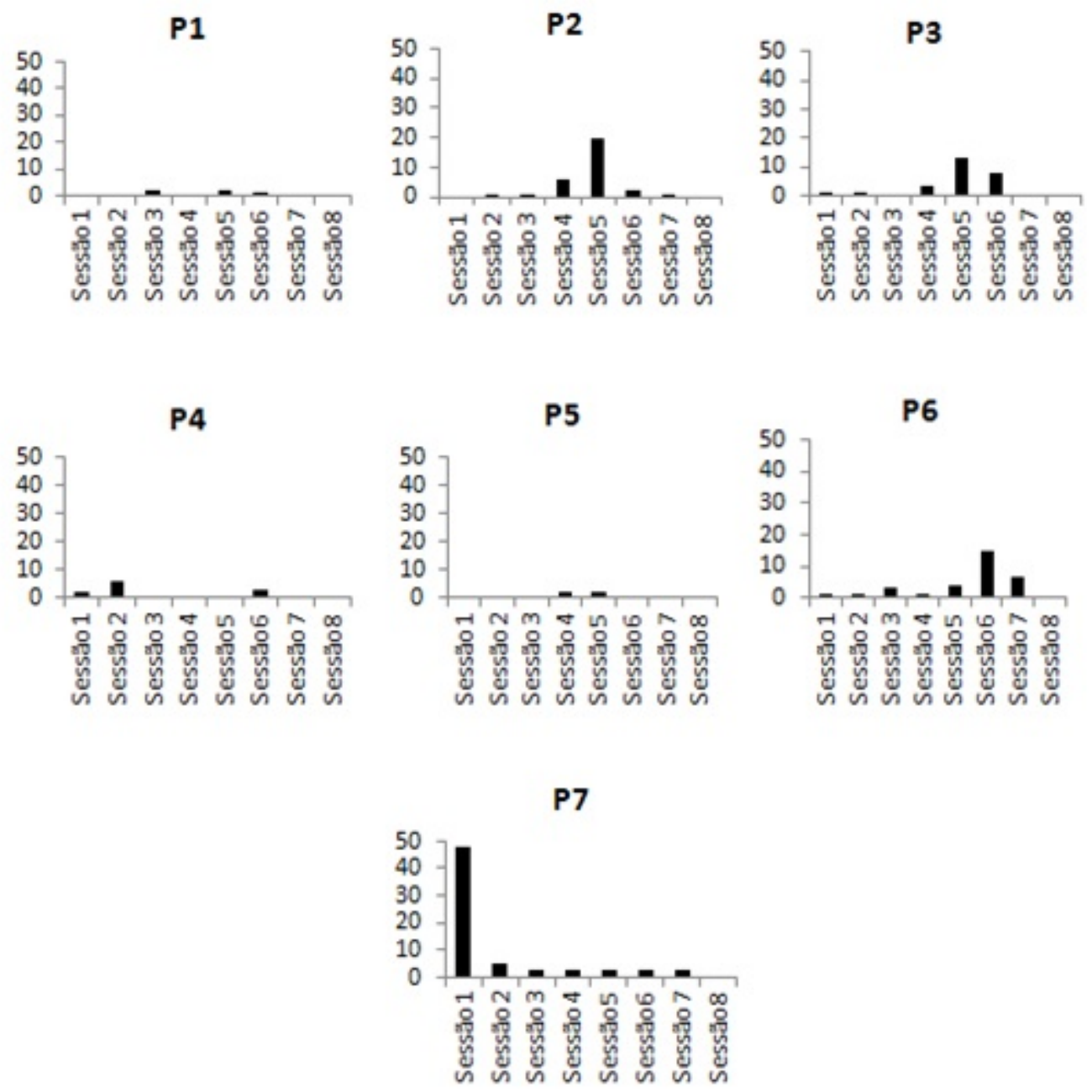

Figura 2. Frequência de problemas de comportamento apresentados por cada um dos participantes em cada uma das sessões de intervenção.

Somente os participantes P5 e P6 frequentaram todas as sessões de intervenção. P1 e P4 faltaram a dois encontros e os demais participantes faltaram a um encontro. Quanto as habilidades sociais os participantes tiveram aumento na frequência a partir da sessão 4 - que se manteve até a última sessão de intervenção à qual participaram. P6 apresentou um aumento considerável na frequência de habilidades sociais na última sessão de intervenção, na qual somente ele e P5 estiveram presentes. P7 foi o participante que apresentou as frequências mais baixas de habilidades sociais durante as sessões de intervenção, mas que ficaram na casa de 50 ocorrências, enquanto que seus problemas de comportamento estavam em frequência 5, durante as sessões. Quanto aos problemas de comportamento P1, P4 e P5 apresentaram frequências muito baixas durante as sessões de intervenção. O P7 apresentou alta frequência de problemas de comportamento na sessão inicial e a partir da segunda sessão as frequências foram baixas. P2, P3 e P6 
apresentaram uma frequência alta de problemas de comportamento em uma das sessões de intervenção e baixa frequência nas demais.

\section{Discussão}

Pelos resultados pode-se observar que apesar de todos os participantes não terem deixado os níveis clínicos para os comportamentos externalizantes, internalizantes e totais, os comportamentos externalizantes apresentaram diferença significativa estatística quando comparadas as fases de pré e pós-teste a partir dos instrumentos $C B C L$ e TRF, ou seja, que pais e professores relataram que esses problemas de comportamento reduziram de frequência nos ambientes familiar e escolar após a intervenção em habilidades sociais. O que reforça a intervenção em habilidades sociais na infância como forma de reduzir problemas de comportamento (Kim et al., 2010; Webster-Stratton, Reid, \& Hamond, 2001).

A partir das análises dos comportamentos apresentados durante as sessões de intervenção em análises sociais pôde-se verificar que a frequência dos comportamentos problemas se manteve baixa durante as sessões de intervenção para todos os participantes. Isso provavelmente se deve ao fato de o ambiente terapêutico ter controlado a emissão de problemas de comportamento a partir da valorização e ensino das habilidades sociais (Herman et al., 2011; $\mathrm{Kim}$ et al., 2010). As habilidades sociais eram consequenciadas com o reforço positivo contingente e, assim pode-se se supor que as habilidades sociais funcionaram como respostas funcionalmente equivalentes (Bolsoni-Silva \& Carrara, 2010; Goldiamond, $2002 / 1974)$ aos problemas de comportamento, que perderam sua função de obter atenção e resolver problemas (Maravieski \& Serralta, 2011; Wielewichi, 2011), manteve-se baixa no decorrer dos encontros, ou seja, à medida que os comportamentos habilidosos acontecem com alta frequência os problemas de comportamento tendem a acontecer em baixa frequência por assumirem a mesma função. Por outro lado, as habilidades sociais ocorreram com maior frequência desde a primeira sessão e aumentaram com o passar das sessões de intervenção, demonstrando que o treino foi eficaz (Cox, 2005; Scott, Knapp, Henderson, \& Maughan, 2001) e que generalizou para os contextos familiar e escolar (Elias et al., 2012; Gonçalves \& Murta, 2008; Salvo et al., 2005; Vettorazzi et al., 2005). No entanto, o presente estudo apresentou a redução de problemas de comportamento e aumento na frequência de habilidades sociais com um número reduzido de sessões (oito) o que é esperado pela Psicologia Baseada em Evidências (APA, 2006) para garantir a eficácia de um estudo. É importante facilitar um repertório com alta 
frequência de habilidades sociais e os manter nos diversos ambientes em que a criança está inserida, para que os comportamentos problema ocorram com nenhuma ou com baixa frequência como apontado pelos instrumentos CBL e TRF.

A maioria das pesquisas brasileiras com intervenção em habilidades sociais envolve a participação de pais (Coelho \& Murta, 2007; Kanamota, 2013; Lambertucci \& Carvalho, 2008; Orti, Bolsoni-Silva, Grecco \& Matsunaka, 2015; Orti, Bolsoni-Silva, \& Villa, 2015; Pinheiro, Haase, A. Del Prette, Amarante, \& Del Prette, 2006) ou pais e crianças (Gonçalves \& Murta, 2008; Salvo, Mazzarotto, \& Lörh, 2005). As intervenções em habilidades sociais que têm como participantes somente crianças tem como foco um grupo de habilidades específicas (Borges \& Marturano, 2003; Elias, Marturano, \& Motta-Oliveira, 2012; Vettorazzi et al., 2005 ); além de envolver maior quantidade de sessões (Elias, Marturano, \& Motta-Oliveira, 2012; Vettorazzi et al., 2005); a variável linha de base e seguimento para a verificação da manutenção dos comportamentos aprendidos após o término do procedimento de intervenção não foi encontrada nos estudos selecionados; poucos estudos avaliaram os participantes em dois ambientes diferentes, como escola e família (Lopes, Del Prette et al., 2013), além de poucas apresentarem medidas de produto e processo da intervenção (Borges \& Marturano, 2003; Castro, Melo, \& Silvares, 2003; Lopes et al., 2013).

O presente estudo incluiu a avaliação dos comportamentos apresentados ao longo do processo de intervenção (Tourinho et al., 2007; Wampold et al., 2007), sendo outro aspecto a ser destacado, pois a maioria das pesquisas de intervenção com crianças valoriza a avaliação do desempenho com instrumentos pré e pós intervenção (Elias, Marturano, \& Motta-Oliveira, 2012; Gonçalves \& Murta, 2008; Vettorazzi et al., 2005) e a minoria das pesquisas apontaram mudanças dos participantes ao longo da intervenção (Borges \& Marturano, 2003; Castro, Melo, \& Silvares, 2003; Lopes et al., 2013). Os participantes da intervenção em habilidades sociais foram crianças diagnosticadas com problemas de comportamento internalizantes, externalizantes e totais nos ambientes escolar e familiar, portanto, são crianças que mantém um repertório de problemas de comportamento altamente elevado nos dois ambientes que frequentam. O fato de essas crianças terem apresentado baixos níveis de problemas de comportamento durante as sessões mostra que em um ambiente lúdico, em que elas tenham a possibilidade de se expressar e de receber atenção positiva, valendo-se de habilidades sociais, por exemplo, elas não precisam apresentar problemas de comportamento, ou seja, o problema de comportamento perde a função nesse ambiente (Bolsoni-Silva \& Carrara, 2010). Esse também é um ponto forte do estudo, que o diferencia dos estudos apresentados pela literatura (Elias, Marturano, \& Motta-Oliveira, 
2012; Vettorazzi et al., 2005), já que envolveu participantes que apresentavam repertório clínico bem instalado (ambiente escolar e familiar) para todos os problemas de comportamento apontados anteriormente e, assim mesmo, apontou melhoras nos repertórios do ponto de vista de pais e professores. Nesse sentido o estudo contemplou aspectos importantes para a psicologia preventiva (Oliveira, 2012; Scott et al., 2001) e a psicologia baseada em evidência (APA, 2006). Visto que promoveu uma intervenção simultaneamente eficaz (que atinjam os objetivos iniciais) e efetiva (de curto prazo e com baixos custos) (Cox, 2005) a partir do tratamento de problemas presentes, com um número reduzido de sessões e no ambiente escolar, podendo favorecer a aplicação em grande escala por psicólogos escolares.

De acordo com os resultados dos instrumentos respondidos por pais e professores ( $\mathrm{CBCL}$ e TRF) os comportamentos internalizantes não apresentaram diferença estatística com significância quando comparadas as fases de intervenção (linha de base-pré-teste, préteste-pós-teste, pós-teste-seguimento), no entanto, a partir da observação da interação criança-pais os comportamentos depressivos (internalizantes) passarem de $86,89 \%$ na fase inicial (sessões 1 e 2) para $36,37 \%$ na fase final (sessões 6,7 e 8 ). Isso pode ser devido a atenção exclusiva recebida pela criança durante a brincadeira e por este ter sido um ambiente altamente positivo. A pesquisadora não teve como controlar a atenção recebida pela criança nos ambientes escolar e familiar. Mesmo apresentando uma redução visível dos comportamentos internalizantes quando a relação foi em um ambiente controlado, pais e professores não relataram mudança destes comportamentos nos ambientes escolar e familiar, portanto possíveis estratégias devem ser tomadas para que esses comportamentos deixem de fazer parte do repertório das crianças também em ambientes não controlados. Algumas estratégias podem ser, role-playing de comportamentos concorrentes, facilitação da exposição das crianças durante as atividades, incentivo e reforço positivo da fala das crianças durante as sessões de intervenção.

Como os demais ambientes sociais nos quais a criança está inserida nem sempre dispõe desse mesmo controle, uma dificuldade que pode ser encontrada é o de garantir que os comportamentos aprendidos durante a intervenção sejam generalizados para os ambientes não terapêuticos, no entanto, a partir dos instrumentos CBCL e TRF podese verificar que a frequência dos problemas de comportamentos externalizantes e internalizantes diminuiu com diferença estatisticamente significativa. Outra variável do estudo que garantiu afirmar que a intervenção foi capaz de modificar os comportamentos dos participantes foi o método priorizado do sujeito sobre controle dele mesmo (Cozby, 2006), no qual os comportamentos do participante são comparados em linha de base, pré-teste, pós-teste e 
seguimento. O que aumenta a probabilidade do resultado ser garantido pela intervenção e não simplesmente que os comportamentos seriam modificados mesmo que o participante não passasse por um grupo de intervenção.

A categoria de habilidades sociais "disponibilidade social e cooperação" abrangia o maior número de comportamentos dentre todas as categorias e foram os comportamentos mais apresentados pelos participantes, pois após busca nos estudos que diferenciavam os comportamentos de crianças clínicas e não clínicas para problemas de comportamento, as habilidades que foram apontadas como diferenciadoras dos repertórios dessas crianças foram dessa categoria. Possivelmente porque se tratavam de habilidades que eram frequentemente reforçadas como 'assistir ao filme quieto', 'participar das atividades', 'conversar', 'dar a opinião', 'prestar atenção' e que poderiam ser emitidos durante todo o tempo ao longo das sessões, em comparação com os comportamentos das outras categorias como, por exemplo, o comportamento de cumprimentar da categoria civilidade, que pode ser apresentado uma vez ao início e outra ao final das sessões. Isso se deveu ao fato de que após a busca pela literatura (Barham \& Cia, 2009; Silva, Marturano, Pereira, \& Mafrinato, 2006; Leme \& Bolsoni-Silva, 2010; Elias, Marturano, \& Motta-Oliveira, 2012).

O reforço diferencial apresentado pela aplicadora durante as sessões pode ter influenciado a mudança nas frequências de comportamento. O ambiente terapêutico altamente reforçador, assim como os antecedentes aos comportamentos dos participantes possivelmente tiveram relação com os comportamentos apresentados.

Apesar das mudanças observadas ao longo das sessões e a partir do relato de pais e professores é importante ressaltar a dificuldade encontrada em manter a frequência da presença dos participantes em todas as sessões de intervenção em habilidades sociais, apesar das sessões serem realizados na escola das crianças, sendo, portanto, de fácil acesso. Nota-se que apenas dois dos participantes estiveram presentes em todas as sessões de intervenção, o que pode ter influência sobre os resultados encontrados, principalmente quando se utilizou a somatória das frequências dos participantes para se obter a média das frequências de comportamentos apresentados ao longo das sessões, já que todas as faltas se deram nas últimas sessões (sessões 7 e 8). O fato das sessões ocorrerem no contra-turno pode ter prejudicado a adesão, o ideal é obter o apoio da escola para a intervenção ocorrer no horário de aula, realizado de forma a não trazer prejuízo. 


\section{Considerações finais}

O procedimento foi capaz de ensinar habilidades sociais para crianças que apresentavam problemas de comportamento internalizantes, externalizantes e totais nos ambientes escolar e familiar. Destacamse alguns pontos fortes do estudo: (a) seleção, para treino, de habilidades sociais que diferenciavam o repertório de crianças clínicas e não clínicas para problemas de comportamento a partir de revisão de estudos empíricos; (b) realização de uma intervenção com reduzido número de sessões (oito); (c) o atendimento exclusivo às crianças, que apresentavam problemas de comportamento internalizantes e externalizantes em dois ambientes distintos, pois foram selecionadas as crianças com mais problemas de comportamento, em comparação com outras que poderiam apresentar problemas apenas na escola ou apenas na família; (d) delineamento experimental de sujeito único com sete participantes, incluindo medidas de linha de base, pré e pós-testes e seguimento; condução da intervenção na escola, facilitando a aplicação, o que poderia ser realizado em grande escala, via política pública.

Uma limitação do estudo foi não realizar uma sessão de seguimento com os participantes na qual pudessem ser verificados quais dos comportamentos apresentados pelos mesmos durante as sessões de intervenção em habilidades sociais foram mantidos seis meses após o término da intervenção na relação Participante-Participante e Participante-Terapeuta. Os dados referentes ao seguimento apresentavam apenas o relato de pais (CBCL) e professores (TRF).

Outra limitação do estudo foi a de não apresentar os dados referentes a avaliação de pais e professores quanto aos comportamentos habilidosos dos Participantes. No presente estudo foram avaliados pelos mesmos apenas os dados referentes aos problemas de comportamento dos Participantes.

Estudos futuros podem ampliar a aplicação do procedimento com um número maior de participantes, considerando delineamento experimental de grupo e/ou delineamento de sujeito único que controle a variável tempo, como em delineamento de linha de base múltipla. No que se refere às medidas de processo, sugere-se 0 estudo da interação terapeuta-clientes.

\section{Referências}

Achenbach, T. M., \& Edelbrock, C. S. (1979). The Child Behavior Profile: II. Boys aged 12-16 and girls aged 6-11 and 12-16. Journal of consulting and clinical psychology, 47(2), 223.

Achenbach, T. M. \& Rescorla, L. (2001). ASEBA School-Age Forms \& Profiles. Aseba. 
American Psychological Association (2006). Evidence-Based Practice In: Psychology. American Psychologist, 61(4), 271-285.

Barham, E. J., \& Cia, F. (2009). Repertório de habilidades sociais, problemas de comportamento, autoconceito e desempenho acadêmico de crianças no início da escolarização, Estudos de Psicologia, 26(1), 45-55.

Berry, D., \& O'Connor, E. (2010). Behavioral risk, teacher-child relationships, and social skill development across middle childhood: A child-by-environment analysis of change. Journal of Applied Developmental Psychology, 31(1), 1-14.

Bolsoni-Silva, A. T., \& Carrara, K. (2010). Habilidades sociais e análise do comportamento:compatibilidades e dissensões conceitual-metodológicas. Psicologia em Revista, Belo Horizonte, 16(2), 330-350.

Bolsoni-Silva, A. T., \& Loureiro, S. R. (2011). Práticas educativas parentais e repoertório comportamental infantil: comparando crianças diferenciadas pelo comportamento. Paidéia, 21 (48), 61-71.

Bolsoni-Silva, A. T., Marturano, E. M., \& Loureiro, S. R. (2009). Construction and validation of the brazilian Questionario de Respostas Socialmente Habilidosas segundo relato de professores (QRSH-PR). Spanish Journal of Psychology, 12(1), 349-359.

Bolsoni-Silva, A. T., Marturano, E. M., \& Loureiro, S. R. (2011). Estudos de Confiabilidade e Validade do Questionário de Respostas Socialmente Habilidosas Versão para Pais-QRSHPais. Psicologia: Reflexão e Crítica, 24(2), 227-235.

Bolsoni-Silva, A. T., Marturano, E. M., Pereira, V. A., \& Manfrinato, J. D. S. (2006). Habilidades sociais e problemas de comportamento de pré-escolares: comparando avaliações de mães e de professoras. Psicologia: Reflexão e Crítica, 19(3), 460-469.

Bolsoni-Silva, A.T., Paiva, M.M., \& Barbosa, C.G. (2009). Problemas de Comportamento de crianças/adolescentes e dificuldade de pais/cuidadores: um estudo de caracterização. Psicologia Clínica, 21(1), 169-184.

Bolsoni-Silva, A. T., Villas Boas, A. C. V. B., Leme, V. B. R., \& Silveira, F. F. (2010). Caracterização de programas de intervenção com crianças e/ou adolescentes. Arquivos Brasileiros de Psicologia, 62(1), 104-114.

Borges, D. S. C., \& Marturano, E. M. (2003). Desenvolvendo habilidades de solução de problemas interpessoais no ensino fundamental. Paidéia: Cadernos de Psicologia e Educação, 12, 120-129. 
Branco, C. M., \& Ferreira, E. A. P. (2006). Descrição do atendimento de uma criança com déficit em habilidades sociais. Revista Brasileira de Terapia Comportamental e Cognitiva, 8(1), 25-38.

Castro, R. E. F., Melo, M. H. S., \& Silvares, E. F. M. (2003). O julgamento por pares de crianças com dificuldades interativas após um modelo ampliado de intervenção. Psicologia: Reflexão e Crítica, 16(2), 309-318.

Coelho, M. V., \& Murta, S. G. (2007). Treinamento de pais em grupo: um relato de experiência. Estudos de psicologia, 24(3), 333341.

Comodo, C. N., Del Prette, A., Del Prette, Z. A. P., \& Manólio, C. L. (2011). O passeio de Bia (vídeo): apresentação e validade interna e externa de um recurso para a promoção de habilidades sociais de pré-escolares. Psicologia: Teoria e Prática, 13(1), 34-47.

Cox, D. D. (2005). Evidence-Based Interventions Using Home Collaboration. School Psychology Quarterly, 20(4), 473-497.

Cozby, P. C. (2006). Métodos de pesquisa em ciências do comportamento. São Paulo: Atlas.

Freitas, L. C., Oliveira-Barreto, S., \& Del Prette, Z. A. P., (2011). Habilidades sociais na comorbidade entre dificuldades de aprendizagem e problemas de comportamento: Uma avaliação multimodal, Psico, 42(4), 503-510.

Del Prette, A., \& Del Prette, Z. A. P. (2005). Sistema Multimídia de Habilidades Sociais para Crianças. São Paulo, SP: Casa do Psicólogo.

Elias, L. C. S., Marturano, E. M., \& Motta-Oliveira, A. M. A. (2012). Eu posso resolver problemas: um programa para 0 desenvolvimento de habilidades de solução de problemas interpessoais. Temas em Psicologia, 20(2), 521-535.

Fagundes, A. J. F. M. (1999). Descrição, definição e registro de comportamento. São Paulo: Edicon.

Farmer, T. W., \& Xie, H. (2007). Aggression and school social dynamics: The good, the bad, and the ordinary. Journal of School Psychology, 45(5), 461-478.

Goldiamond (2002/1974). Toward a constructional approach to social problems: ethical and constitucional issues raised by Applied Behavior Analysis. Behavior and Social Issues, 11, 108-197 (originalmente publicado em 1974), Behaviorism, 2, 1-84.

Gonçalves, E. S., \& Murta, G, S. (2008). Avaliação dos Efeitos de uma Modalidade de Treinamento de Habilidades Sociais para Crianças. Psicologia: Reflexão e Crítica, 21(3), 430-436.

Herman, K. C., Borden, L. A., Reinke, W. M., \& Webster-Stratton, C. (2011). The Impact of the Incredible Years Parent, Child, and Teacher Training Programs on Children's Co-Occurring 
Internalizing Symptoms. School Psychology Quarterly, 26(3), 189-201.

Kanamota, P. F. C. (2013). Estudo da influência das respostas de empatia e recomendação do terapeuta na interação terapeutacliente e descrição de efeitos de um procedimento de intervenção para o tratamento de mães de adolescentes com problemas de comportamento. Dissertação de mestrado, Universidade Estadual Paulista, Bauru, SP, Brasil.

Kim, M. J., Doh, H. S, Hong, J. S, \& Choi, M. K. (2010). Social skills training and parent education programs for aggressive preschoolers and their parents in South Korea. Children and Youth Services Review, 33, 838-845.

Lambertucci, M. R., \& Carvalho, H. W. D. (2008). Avaliação da efetividade terapêutica de um programa de treinamento de pais em uma comunidade carente de Belo Horizonte. Contextos Clínicos, 1(2), 106-112.

Leme, V. B., \& Bolsoni-Silva, A. T. (2010a). Habilidades Sociais Educativas Parentais e comportamentos de pré-escolares. Estudos de Psicologia, 15(2), 161-173.

Leme, V. B., \& Bolsoni-Silva, A. T. (2010b). Habilidades sociais e problemas de comportamento: um estudo exploratório baseado no modelo construcional. Aletheia, 31(1), 149-167.

Lopes, D. C., Del Prette, Z. A. P., \& Del Prette, A. (2013). Recursos multimedia no ensino de habilidades sociais a crianças de baixo rendimento acadêmico. Psicologia: Reflexão e Crítica, 26(3), 451-458.

Machado, L. D., Dahl, C. M., Carvalho, M. C. C., \& Cavalcanti, M. T. (2007). Programa de tratamento assertivo na comunidade (PACT) e gerenciamento de casos (case management): revisão de 20 anos da literatura. Jornal Brasileiro de Psiquiatria, 56(3), 208-218.

Maravieski, S., \& Serralta, F. B. (2011). Características clínicas e sociodemográficas da clientela atendida em uma clínica-escola de Psicologia. Temas em Psicologia, 19(2), 481-490.

Oliveira, S. A. (2012). Prevenção em saúde mental no Brasil na perspectiva da literatura e de especialistas da área. Dissertação de mestrado, Universidade de Brasília, Brasília, DF, Brasil.

Orti, N. P., Bolsoni-Silva, A. T., Grecco, M. K., \& Matsunaka, M. P. S. (2015). Parent Intervention With Mothers Of Children With Internalizing Problems:Analysis Of Complaints, Themes And Therapist-Client Interaction In Three Clinical Cases. Journal of Psychological Abnormalities in Children, 4, 139. doi: 10.4172/2329-9525.1000139.

Orti, N. P, Bolsoni-Silva, A. T., \& Villa, M. B. (2015). Assessment of the Effects of a Parental Intervention with Mothers of Children 
with Internalizing Problems. Advances in Research, 4(5), 279292.

Pinheiro, M. I. S., Haase, V. G., Del Prette, A., Amarante, C. L. D., \& Del Prette, Z. A. P. (2006). Treinamento de habilidades sociais educativas para pais de crianças com problemas de comportamento. Psicologia: Reflexão e Crítica, 19(3), 407-414.

Salvo, C. G., Mazzarotto, I. H. K., \& Löhr, S, S. (2005). Promoção de habilidades sociais em pré-escolares. Revista Brasileira Crescimento Desenvolvimento Humano, 15(1), 46-55.

Scott, S., Knapp M., Henderson J., \& Maughan, B. (2001). Financial cost of social exclusion: Follow up study of antisocial children into adulthood. British Medical Journal, 323, 191-194.

Scott, S., Knapp M., Henderson J., \& Maughan, B. (2001). Financial cost of social exclusion: Follow up study of antisocial children into adulthood. British Medical Journal, 323, 191-194.

Tinker Bell e o Tesouro Perdido. (2009). Direção: Bradley Raymond. Roteirista: Margot Pipkin. Animação. 80 minutos.

Tourinho, E. Z., Neno, S., Batista, J. R., Garcia, M. G., Brandão, G. G., Souza, L. M., \& Oliveira-Silva, M. (2007). Condições de treino e sistemas de categorização de verbalizações de terapeutas. Revista Brasileira de Terapia Comportamental e Cognitiva, 9(2), 317-336.

Wampold, B. E., Goodheart, C. D., \& Levant, R. F. (2007). Clarification and elaboration on evidence-based practice in psychology. American Psychologist, 62(6), 616-618.

Webster-Stratton, C., Reid, J., \& Hamond, M. (2001). Social Skills and Problem-solving Training for Children with Early-onset Conduct Problems: Who Benefits? Journal of Child Psychology and Psychiatry, 42(7), 943-952.

Weisz, J. R., Sandler, I. N., Durlak, J. A., \& Anton, B. S. (2005). Promoting and protecting youth mental health through evidence-based prevention and treatment. American Psychologist, 60, 628-648.

Wielewicki, A. (2011). Problemas de comportamento infantil: importância e limitações de estudos de caracterização em clínicas-escola brasileiras. Temas em Psicologia, 19(2), 379389.

\section{Endereço para correspondência \\ Alessandra Pereira Falcão}

Universidade Estadual Paulista - Julio de Mesquita Filho / Campus Bauru - SP

Av. Eng. Luiz Edmundo Carrijo Coube, 14-01, Vargem Limpa, CEP 17033-360,

Bauru - SP, Brasil

Endereço eletrônico: alepfalcao@hotmail.com

Alessandra Turini Bolsoni-Silva

Universidade Estadual Paulista - Julio de Mesquita Filho / Campus Bauru - SP 
Av. Eng. Luiz Edmundo Carrijo Coube, 14-01, Vargem Limpa, CEP 17033-360, Bauru - SP, Brasil

Endereço eletrônico: bolsoni@fc.unesp.br

\section{Natália Magri}

Universidade Estadual Paulista - Julio de Mesquita Filho / Campus Bauru - SP

Av. Eng. Luiz Edmundo Carrijo Coube, 14-01, Vargem Limpa, CEP 17033-360, Bauru - SP, Brasil

Endereço eletrônico: natmagri@gmail.com

Laísa Aparecida Moretto

Universidade Estadual Paulista - Julio de Mesquita Filho / Campus Bauru - SP

Av. Eng. Luiz Edmundo Carrijo Coube, 14-01, Vargem Limpa, CEP 17033-360,

Bauru - SP, Brasil

Endereço eletrônico: laisamoretto@hotmail.com

Recebido em: 07/07/2015

Reformulado em: 20/01/2016

Aceito para reformulação em: 30/03/2016

\section{Notas}

* Psicóloga, Mestre e doutoranda em Psicologia do desenvolvimento e aprendizagem pela Unesp/Bauru. Sócia-proprietária do Centro de Psicologia Aplicada-Psicovida em Londrina-PR onde atua como psicóloga clínica. Docente no curso de Psicologia na Universidade Filadélfia - Unifil em Londrina-PR.

** Psicóloga, Professora Adjunta da Faculdade de Ciências de Bauru/ Unesp e docente de Psicologia e do Curso de Pós-Graduação em Psicologia do Desenvolvimento e Aprendizagem, na Universidade Estadual Paulista/ Bauru. Coordena o Serviço de Avaliação e Intervenção junto a pais, casais, crianças e universitários, junto ao Centro de Psicologia Aplicada da Unesp - Bauru e Laboratório de Aprendizagem, Desenvolvimento e Saúde - LADS.

*** Psicóloga. Na graduação atuou como pesquisadora do Grupo de Estudos e Pesquisa na área de comportamentos pró sociais e problemas de comportamentos infantis. Desenvolveu atividades de Pesquisa e Iniciação Científica na área de Habilidades sociais infantis e Práticas Parentais.

**** Psicóloga, Mestranda em Psicologia do desenvolvimento e aprendizagem pela Unesp/Bauru. Foi bolsista de Iniciação científica pelo conselho Nacional de Desenvolvimento Científico e Tecnológico ( $\mathrm{CNPq})$, em que desenvolveu atividades de pesquisa em tratamento e prevenção psicológica sobre problemas de comportamento infantis.

\section{Apoio financeiro: CAPES - bolsa de mestrado da primeira autora e iniciação científica das terceira e quarta autoras.}

